\title{
INVARIANT DENSITY ESTIMATION FOR A REFLECTED DIFFUSION USING AN EULER SCHEME.
}

\author{
PATRICK CATTIAUX * , JOSÉ R. LEÓN * , AND CLÉMENTINE PRIEUR ${ }^{\circ}$ \\ - Institut de Mathématiques de Toulouse \\ * Escuela de Matemática, Caracas \\ $\odot$ Laboratoire Jean Kuntzmann, Grenoble
}

\begin{abstract}
We give an explicit error bound between the invariant density of an elliptic reflected diffusion in a smooth compact domain and the kernel estimator built on the symmetric Euler scheme introduced in Bossy, Gobet and Talay (2004).
\end{abstract}

Key words : ergodic reflected diffusion process; Euler scheme rate of convergence for large times; discrete approximation for the invariant measure.

$M S C 2010$

Primary: 60J60.

Secondary: 60H10 Stochastic ordinary differential equations; 37TL40 Invariant measures; 37A25 Ergodicity, mixing, rates of mixing.

\section{Introduction and main results.}

Let $D$ be a bounded, open domain (hence connected) in $\mathbb{R}^{d}$ with a smooth (say $C^{\infty}$ ) boundary $\partial D$. We denote by $\bar{D}$ the closure of $D$ and for $x \in \partial D$ we introduce $n(x)$ the unit normal vector to $\partial D$ at $x$ pointing inward.

Let $\sigma$ be some $C^{\infty}(\bar{D})(d, m)$ matrices field, $b$ and $V$ be $C^{\infty}(\bar{D})$ vectors field. We assume that

$$
\begin{aligned}
a(x)=\sigma(x) \sigma^{*}(x) & \geq a I d \text { for some } a>0 \text { and all } x \in \bar{D}, \text { uniform ellipticity, } \\
V(x) \cdot n(x) & \geq v \text { for some } v>0 \text { and all } x \in \partial D, \text { reflection. }
\end{aligned}
$$

We then consider the associated diffusion process $X$. with oblique reflection on $\partial D$, i.e. the solution of the following system of stochastic differential equations with reflection

$$
\left\{\begin{array}{l}
d X_{t}=\sigma\left(X_{t}\right) d W_{t}+b\left(X_{t}\right) d t+V\left(X_{t}\right) d L_{t} \quad \text { and } \quad X_{0}=x \in \bar{D} \\
L_{t}=\int_{0}^{t} \mathbb{I}_{X_{s} \in \partial D} d L_{s} \quad \text { and } \quad \int_{0}^{t} \mathbb{1}_{X_{s} \in \partial D} d s=0
\end{array}\right.
$$

In other words, if

$$
\mathcal{L}=\frac{1}{2} \sum_{j, k=1}^{d} a_{j, k}(x) \partial_{j, k}^{2}+\sum_{j=1}^{d} b_{j}(x) \partial_{j}
$$

Date: March 13, 2017. 
then for all $f \in C^{\infty}(\bar{D})$ such that

$$
V f(x)=\sum_{j=1}^{d} V_{j}(x) \partial_{j} f(x)=0 \quad \text { for all } x \in \partial D,
$$

the process $t \mapsto f\left(X_{t}\right)-f(x)-\int_{0}^{t} \mathcal{L} f\left(X_{s}\right) d s$ is a martingale.

Existence and uniqueness of a solution is well known (see e.g. Ikeda and Watanabe (1981)). Denote by $\mathbb{P}_{x}$ the law of the solution (here we mean the law of $X$.). Then on the paths space $C^{0}\left(\mathbb{R}^{+}, \bar{D}\right)$ equipped with the standard filtration, the family $\left(\mathbb{P}_{x}\right)_{x \in \bar{D}}$ is a strong Markov family. We can then define on $\mathbb{L}^{\infty}(\bar{D})$ the semi-group

$$
P_{t} f(x)=\mathbb{E}_{x}\left[f\left(X_{t}\right)\right] .
$$

Actually this semi-group is strong Feller, i.e. is mapping $\mathbb{L}^{\infty}$ into $C^{0}(\bar{D})$ and its generator coincides with $\mathcal{L}$ on the set of $f \in C^{\infty}(\bar{D})$ satisfying (1.4).

Much more is known. Using results in partial differential equations or using Malliavin calculus and some large deviations arguments, one can show the following

Proposition 1.5. For all $t>0, P_{t} f(x)=\int_{\bar{D}} f(y) p_{t}(x, y) d y$ where $p$. is continuous from ] $0,+\infty\left[\right.$ into $C^{\infty}(\bar{D} \times \bar{D})$.

In addition for all $t>0$,

$$
\inf _{(x, y) \in \bar{D} \times \bar{D}} p_{t}(x, y)=\alpha_{t}>0 .
$$

For all this and what follows we refer to Cattiaux (1992), in particular Section 2 for the existence of smooth density kernels, and Section 5 for strict positivity using a smart large deviations argument. The results in Cattiaux (1992) include some elliptic degenerate situations. We shall explain later why we only consider here the non degenerate situation.

(1.6) allows to prove the existence and uniqueness of an invariant probability measure $\pi$ for the process (see Section 5 in Cattiaux (1992)). Once again it is easily seen from the ellipticity assumption (1.1) that

$$
\pi(d y)=p_{i n v}(y) d y \quad \text { where } p_{i n v} \in C^{\infty}(\bar{D}) \text { and } \inf _{y \in \bar{D}} p_{i n v}(y)>0 .
$$

Our goal in this paper is to provide a pointwise estimate of $p_{\text {inv }}(y)$ for some $y \in D$.

Of course using Green's formula, $\pi$ satisfies some boundary value problem

$$
\begin{aligned}
\mathcal{L}^{*} \pi=0 & \text { in } D, \\
V^{*} \pi=0 & \text { on } \partial D,
\end{aligned}
$$

where $\mathcal{L}^{*}$ is the adjoint of $\mathcal{L}$ and $V^{*}$ the adjoint of $V$ with respect to the co-normal derivative (see Cattiaux (1992) p. 605 for the explicit calculations). So one can use numerical schemes to get approximate values for $p_{i n v}$. These schemes can be more or less efficient. 
Here we propose an alternative stochastic method, combining a kernel estimator and some Euler scheme. More precisely, consider the symmetrized Euler scheme $X^{h}$ introduced by Bossy et al. (2004), we shall describe in details in the next section and the estimator

$$
\hat{p}^{n, h}(y)=\frac{1}{n b_{n}^{d}} \sum_{i=1}^{n} K\left(\frac{y-X_{i h}^{h}}{b_{n}}\right)
$$

for some non-negative smooth kernel $K$ compactly supported in a neighborhood of the origin and such that $\int K(z) d z=1$. We shall get a bound for the quadratic error

$$
\mathbb{E}_{x}\left(\left|\hat{p}^{n, h}(y)-p_{\text {inv }}(y)\right|^{2}\right)
$$

in terms of $n, h$ and the choice of the bandwidth $b_{n}$.

At the same time we obtain a similar result for the error evaluation in the estimation of an observable. For some smooth function $f$ let us consider $\pi(f)=\int f d \pi$. The practical evaluation of $\pi(f)$ through the symmetric Euler scheme is defined as

$$
\hat{\pi}_{n}^{h}(f)=\frac{1}{n} \sum_{i=1}^{n} f\left(X_{i h}^{h}\right) .
$$

In the sequel we shall get a bound for the quadratic error

$$
\sup _{x \in \bar{D}} \mathbb{E}_{x}\left[\left|\hat{\pi}_{n}^{h}(f)-\pi(f)\right|^{2}\right] \text {. }
$$

Up to our knowledge, it is the first time that such an estimation for the invariant density is obtained. Indeed, on one hand, there are a lot of papers dealing with the approximation of the density at a finite time $t$ by the one of the corresponding Euler scheme for non reflected diffusion processes (see e.g. Bally and Talay (1996) Gobet and Labart (2008)), and others dealing with the approximation of some observable at finite time like Bossy et al. (2004) for an elliptic reflected diffusion. On the other hand, using Meyn and Tweedie's theory ( Meyn and Tweedie (2009)), some authors obtained approximation results for an observable of the invariant measure using approximation schemes, in particular see Talay (1990), Mattingly, Stuart and Higham (2002) and Shardlow and Stuart (2000).

Here we shall combine finite time approximation results with mixing properties of the process in the spirit of Dedecker and Prieur (2005) and our previous work Cattiaux, Leon and Prieur (2014). Our method extends to non-reflected diffusion processes satisfying some Lyapunov condition as in Mattingly et al. (2002) Shardlow et al. (2000) Talay (1990), and actually the compact situation we are looking at, introduces more intricacies. We decided not to detail the non reflected case to keep this note as short as possible. The theoretical study we conduct hereafter in the compact framework is motivated by some concrete application described in Valera et al. (2016). In that paper, the authors compare several models for the study of the transport of pollutants on water surfaces. In particular, the authors are showing that a reflected diffusion model is promising.

Relaxing the ellipticity assumption (1.1) also introduces some intricacies we shall not discuss here. 


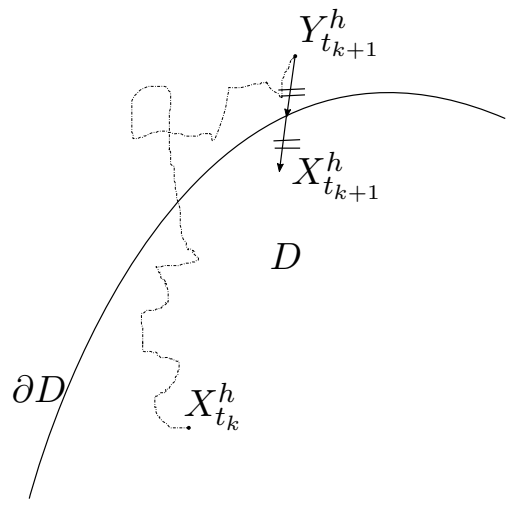

Figure 1. Euler scheme for the reflected diffusion

The paper is organized as follows. Section 2 is devoted to the study of System of Equations (1.3) and of its symmetrized Euler scheme. Long time behaviour is investigated in Section 3. In Section 4 we provide error estimates for kernel estimation procedures based on the symmetrized Euler scheme. In the last section (Section 5), we provide numerical experiments which illustrate the convergence of the kernel density estimator based on the scheme. In this section, we also show the difficulties induced by the tuning of the various parameters involved in the definition of the scheme.

\section{Study of the System And its Symmetrized Euler scheme.}

In Bossy, Gobet and Talay (2004) (after the previous work Gobet (2001a)), the following Euler scheme for the reflected diffusion is proposed (see Figure 1).

For some given $1 \geq h>0$, we introduce the time discretization $t_{k}=k h$, for $k \in \mathbb{N}$. Next we choose some $R>0$ and look at the set $d(y, \bar{D}) \leq R$.

Set $X_{0}^{h}=x$ and for $t_{k} \leq t \leq t_{k+1}$ define

$$
Y_{t}^{h}=X_{t_{k}}^{h}+b\left(X_{t_{k}}^{h}\right)\left(t-t_{k}\right)+\sigma\left(X_{t_{k}}^{h}\right)\left(W_{t}-W_{t_{k}}\right) \text {. }
$$

Finally

(1) if $Y_{t_{k+1}}^{h} \in \bar{D}$, then $X_{t_{k+1}}^{h}=Y_{t_{k+1}}^{h}$.

(2) If $d\left(Y_{t_{k+1}}^{h}, \bar{D}\right)>R$, then $X_{t_{k+1}}^{h}=X_{t_{k}}^{h}$.

(3) If $R \geq d\left(Y_{t_{k+1}}^{h}, \bar{D}\right)>0$, then we choose for $X_{t_{k+1}}^{h}$ the symmetric of $Y_{t_{k+1}}^{h}$ with respect to $\partial D$ in the direction of the reflection vector field $V$. To be sure that this symmetric belongs to $\bar{D}$ we have to choose $R$ small enough, what is assumed from now on. One can remark that this transformation $F$ is smooth (see Gobet (2001a) Appendix for more details.)

For the sequel we shall need a little bit more, namely we need to choose $R$ in such a way that the previous transformation is a $C^{1}$ diffeomorphism from $0<d(y, \bar{D})<R$ to some open subset of $D$. This is also possible. Indeed, first we may choose $R_{y}$ such that $F$ is a local diffeomorphism in the neighborhood $B\left(y, R_{y}\right)$ of any $y \in \partial D$. This is done by choosing a local chart $C_{y}$ at $y$ for which $C_{y} \cap \partial D$ is sent to some open subspace of an hyperplane, and by using the reflexion assumption (1.2) to prove that the Jacobian matrix of $F$ at $y$ is invertible (see 
Gobet (2001a) appendix A), so that the local inverse function theorem applies. Then using compactness one can recover $\partial D$ by a finite number of such local charts $C_{j}$, and show that this covering contains $0<d(y, \bar{D})<R$ for all small enough $R$. Finally it remains to show that $F$ is one to one, and use the global inverse function theorem. To prove that $F$ is one to one on $0<d(y, \bar{D})<R$ when $R$ is small enough, consider a sequence $R_{n}$ going to 0 and a sequence $\left(z_{n}, z_{n}^{\prime}\right) \in\left\{0<d(y, \bar{D})<R_{n}\right\} \times\left\{0<d(y, \bar{D})<R_{n}\right\}$ such that $F\left(z_{n}\right)=F\left(z_{n}^{\prime}\right)$. Using compactness, we may assume that $\left(z_{n}, z_{n}^{\prime}\right)$ converges to some $\left(z, z^{\prime}\right) \in \partial D \times \partial D$. Since $F$ is smooth we get $z=F(z)=\lim _{n} F\left(z_{n}\right)=F\left(z^{\prime}\right)=z^{\prime}$. It follows that $z_{n}$ and $z_{n}^{\prime}$ belong to the same local chart $C_{j}$ for $n$ large enough, so that this contradicts the local diffeomorphism property.

Introduce as before the discrete time semi-group $P_{t}^{h} f(x)=\mathbb{E}\left(f\left(X_{t}^{h}\right) \mid X_{0}^{h}=x\right)$ for $t \in h \mathbb{N}$ and the marginal distribution $P_{t}^{h}(x, d y)$. We clearly have the following Hahn decomposition

$$
P_{t}^{h}(x, d y)=p_{t}^{h}(x, y) d y+r(t, h) \delta_{x} .
$$

Indeed for $k=1$ this distribution is obtained by considering some gaussian random variable restricted to $\bar{D}$ (when $Y_{t_{1}}^{h} \in \bar{D}$ ), or a smooth image (through the diffeomorphism $F$ ) of the same gaussian restricted to $R \geq d(y, \bar{D})>0$, or some Dirac mass at $x$ if some huge increment of the gaussian occurred. Starting from an absolutely continuous distribution, any step furnishes again an absolutely continuous distribution (even if a huge increment occurred since the starting distribution is absolutely continuous). Indeed the singular part is supported by the initial point $x$.

Also notice that there exists some constant $C(b, \sigma, R)$ which is strictly positive provided $h$ is small enough, such that for all $x \in \bar{D}$,

$$
r(k h, h) \leq \exp \left(-\frac{k C(b, \sigma, R)}{h}\right) \sqrt{h}
$$

as the successive increments are independent (conditionally to $X_{t_{k}}^{h}=x$ ) and $r(h, h)$ is less than the probability for a centered gaussian random variable with variance $\left(\max |\sigma|^{2}\right) h$ to be larger than $(R-(\max |b|) h) / d$.

Finally let us recall the main result (Theorem 1) obtained in Bossy, Gobet and Talay (2004)

Theorem 2.2. Under all our assumptions, for $T>1$ there exists some constant $K(T)$ such that for all $N \in \mathbb{N}$ with $N h \leq T$ and all $x \in \bar{D}$,

$$
\sup _{x \in \bar{D}}\left|\mathbb{E}_{x}\left(f\left(X_{N h}^{h}\right)\right)-\mathbb{E}_{x}\left(f\left(X_{N h}\right)\right)\right| \leq K(T) h\|f\|_{C_{b}^{5}(\bar{D})},
$$

where as usual

$$
\|f\|_{C_{b}^{5}(\bar{D})}=\sum_{|\beta| \leq 5} \sup _{z \in \bar{D}}\left|\partial^{\beta} f(z)\right|
$$

We will give some useful explicit bound for $K(T)$,

Proposition 2.3. There exist some constants $\kappa>0$ and $c>0$ such that $K(T)$ in Theorem 2.2 is bounded, i.e. $K(T) \leq c e^{\kappa T}$, uniformly in $h$ for $h$ small enough, for instance $h \leq \frac{1}{4}$.

Proof. Let $N_{h}$ be the unique integer such that $N_{h} h \leq 1<\left(N_{h}+1\right) h$, and define $t_{h}=N_{h} h$. Changing the constant $c$ if necessary it is enough to prove the proposition for $T=N t_{h}$. Indeed 
if $N t_{h} \leq T \leq(N+1) t_{h}$, then $K(T) \leq c e^{\kappa(N+1) t_{h}} \leq c e^{\kappa\left(T+t_{h}\right)} \leq c e^{\kappa} e^{\kappa T}$ since $t_{h} \leq 1$.

Now, using the Markov property

$$
\begin{gathered}
\left|\mathbb{E}_{x}\left(f\left(X_{(N+1) t_{h}}^{h}\right)\right)-\mathbb{E}_{x}\left(f\left(X_{(N+1) t_{h}}\right)\right)\right|= \\
=\left|\int P_{N t_{h}}^{h}(x, d y)\left(\mathbb{E}_{y}\left(f\left(X_{t_{h}}^{h}\right)\right)-\mathbb{E}_{y}\left(f\left(X_{t_{h}}\right)\right)\right)+\int\left(P_{N t_{h}}^{h}(x, d y)-P_{N t_{h}}(x, d y)\right) \mathbb{E}_{y}\left(f\left(X_{t_{h}}\right)\right)\right| \\
\leq \int P_{N t_{h}}^{h}(x, d y)\left|\mathbb{E}_{y}\left(f\left(X_{t_{h}}^{h}\right)\right)-\mathbb{E}_{y}\left(f\left(X_{t_{h}}\right)\right)\right|+\left|\int\left(P_{N t_{h}}^{h}(x, d y)-P_{N t_{h}}(x, d y)\right)\left(\int f(z) p_{t_{h}}(y, z) d z\right)\right| \\
\leq K\left(t_{h}\right) h\|f\|_{C_{b}^{5}(\bar{D})}+K\left(N t_{h}\right) h\left\|\int f(z) p_{t_{h}}(., z) d z\right\|_{C_{b}^{5}(\bar{D})} .
\end{gathered}
$$

Notice that $\frac{1}{2} \leq t_{h} \leq 1$, for all $h \leq \frac{1}{4}$, so that

$$
\left\|\int f(z) p_{t_{h}}(., z) d z\right\|_{C_{b}^{5}(\bar{D})} \leq C\|f\|_{\infty}
$$

for some constant $C$ which only depends on $\sup _{\frac{1}{2} \leq u \leq 1}\left\|p_{u}\right\|_{C_{b}^{5}(\bar{D})}$. A very rough majorization thus yields

$$
\left|\mathbb{E}_{x}\left(f\left(X_{(N+1) t_{h}}^{h}\right)\right)-\mathbb{E}_{x}\left(f\left(X_{(N+1) t_{h}}\right)\right)\right| \leq h K\left(N t_{h}\right)(1+C)\|f\|_{C_{b}^{5}(\bar{D})}
$$

hence the result with $e^{\kappa}=(1+C)^{1 / t_{h_{0}}}$ for some given $h_{0}$, and $c=K(1) e^{\kappa}$ for instance.

The previous proof indicates that we may in a sense improve the control by $\|f\|_{C_{b}^{5}(\bar{D}) \text {. This }}$ kind of improvement can (and will) be of great help, since the previous one only gives some errors for the approximate distribution in a very general space (the dual space of $C^{5}$ functions).

To this end introduce the ordinary diffusion process $Z$. and its Euler scheme $Z^{h}$, associated to the same coefficients $\sigma$ and $b$ (or a $C_{b}^{\infty}$ extension of them outside $\bar{D}$ ). Recall that (see e.g. Bally and Talay (1995)), in this situation we have the existence of an universal constant (only depending on $\bar{D}$ and the coefficients) such that

$$
\left|\mathbb{E}_{x}\left(f\left(Z_{s}^{h}\right)\right)-\mathbb{E}_{x}\left(f\left(Z_{s}\right)\right)\right| \leq C(t) h\|f\|_{\infty}
$$

Only few results are known for the density. According to Bally and Talay (1996) Theorem 2.1 and Corollary 2.1, we also have

Theorem 2.5. Let $f$ be a smooth function compactly supported. Then there exists some $q>0$ such that for $t>0$, there exist positive constants $Q(t)$ and $c(t)$ such that for all $0<s \leq t$ and $h<s d(x$, suppf $) / 2$,

$$
\left|\mathbb{E}_{x}\left(f\left(Z_{s}^{h}\right)\right)-\mathbb{E}_{x}\left(f\left(Z_{s}\right)\right)\right| \leq h \frac{Q(t)}{s^{d q}} \exp \left(-c(t) \frac{d^{2}(x, \text { supp })}{s}\right)\|F\|_{\infty},
$$

where $F$ denotes the distribution function of the (signed measure) $f(z) d z$. Accordingly, if $h \leq$ $c|x-y|$,

$$
\left|q_{s}(x, y)-q_{s}^{h}(x, y)\right| \leq h \frac{Q(t)}{s^{d q}} \exp \left(-c(t) \frac{|x-y|^{2}}{s}\right),
$$

where $q$. and $q^{h}$ denote respectively the densities of $Z$. and $Z^{h}$. 
Actually these results are given in the more general framework of hypo-elliptic diffusions for a modified Euler scheme, but are true for the usual Euler scheme in the uniformly elliptic situation. The power $s^{d q}$ in the pre-factor is improved in Gobet and Labart (2008) and Guyon (2006), and actually under some regularity assumptions is $s^{(d+1) / 2}$.

One can expect to use these results together with well known estimate for hitting times, in order to obtain similar results in the reflected case.

Let us give a first result in this direction

Theorem 2.6. There exist positive constants $C, \theta$ and $c(d)$ such that for all smooth $f$ with compact support included in $D$ and all $T=N h$,

$$
\begin{gathered}
\sup _{x \in \bar{D}}\left|\mathbb{E}_{x}\left(f\left(X_{T}^{h}\right)\right)-\mathbb{E}_{x}\left(f\left(X_{T}\right)\right)\right| \leq \\
\leq C h \ln ^{c(d)}(1 / h)(d(\operatorname{supp} f, \partial D))^{-2 c(d)} \exp \left(\frac{\theta \ln (1 / h) T}{d^{2}(\operatorname{supp} f, \partial D)}\right)\|f\|_{\infty} .
\end{gathered}
$$

Consequently, there exist positive constants $C$ and $\kappa^{\prime}$ such that for all smooth $f$ with compact support included in $D$, all $\varepsilon>\alpha>0$ and all $T=N h$,

$$
\begin{gathered}
\sup _{x \in \bar{D}}\left|\mathbb{E}_{x}\left(f\left(X_{T}^{h}\right)\right)-\mathbb{E}_{x}\left(f\left(X_{T}\right)\right)\right| \leq \\
\leq \frac{C}{\alpha}(d(\operatorname{supp} f, \partial D))^{-2 c(d)}(1+\varepsilon)^{1+c(d)} h^{\frac{\varepsilon-\alpha}{\varepsilon+1}} \exp \left(\frac{\kappa^{\prime}(1+\varepsilon) T}{d^{2}(\operatorname{supp} f, \partial D)}\right)\|f\|_{\infty} .
\end{gathered}
$$

Though the result is written in a very heavy form, the reader will understand in the next section why we are giving such a general form.

Proof. Introduce a small time $s_{h}$ such that $\frac{s_{h}}{h} \in \mathbb{N}^{*}$ to be precisely defined in (2.8). Replacing $t_{h}$ by $s_{h}$ in the previous proof, we have

$$
\begin{gathered}
\left|\mathbb{E}_{x}\left(f\left(X_{(N+1) s_{h}}^{h}\right)\right)-\mathbb{E}_{x}\left(f\left(X_{(N+1) s_{h}}\right)\right)\right|= \\
=\left|\int P_{N s_{h}}^{h}(x, d y)\left(\mathbb{E}_{y}\left(f\left(X_{s_{h}}^{h}\right)\right)-\mathbb{E}_{y}\left(f\left(X_{s_{h}}\right)\right)\right)+\int\left(P_{N s_{h}}^{h}(x, d y)-P_{N s_{h}}(x, d y)\right) \mathbb{E}_{y}\left(f\left(X_{s_{h}}\right)\right)\right| \\
\leq \int P_{N s_{h}}^{h}(x, d y)\left|\mathbb{E}_{y}\left(f\left(X_{s_{h}}^{h}\right)\right)-\mathbb{E}_{y}\left(f\left(X_{s_{h}}\right)\right)\right|+K\left(N s_{h}\right) h\left\|\int f(z) p_{s_{h}}(., z) d z\right\|_{C_{b}^{5}(\bar{D})} .
\end{gathered}
$$

Remark that for the second term we may either get

or

$$
\left\|\int f(z) p_{s_{h}}(., z) d z\right\|_{C_{b}^{5}(\bar{D})} \leq C \sup _{(x, z) \in \bar{D} \times \bar{D}}\left\|p_{s_{h}}(., .)\right\|_{C_{b}^{5}(\bar{D} \times \bar{D})}\|f\|_{\infty},
$$

$$
\left\|\int f(z) p_{s_{h}}(., z) d z\right\|_{C_{b}^{5}(\bar{D})} \leq C \sup _{(x, z) \in \bar{D} \times \bar{D}}\left\|p_{s_{h}}(., .)\right\|_{C_{b}^{6}(\bar{D} \times \bar{D})}\|F\|_{\infty} .
$$

Indeed to control the derivatives w.r.t. $x$, it is enough to differentiate up to five times under the integral sign, and then to integrate by parts one time for introducing $F$. Finally according to Cattiaux (1992) Proposition (2.25) we have for some $q>0$ depending on the data

$$
\sup _{(x, z) \in \bar{D} \times \bar{D}}\left\|p_{s_{h}}(., .)\right\|_{C_{b}^{k}(\bar{D} \times \bar{D})} \leq C\left(1 \wedge s_{h}\right)^{-q\left(k+\frac{d}{2}\right)} .
$$


Actually the result in Cattiaux (1992) is more general (dealing with the hypo-elliptic case). In the elliptic case we think that the right power is less than $k+d / 2$ but we did not find any reference in the P.D.E. literature.

It remains to control $\mathbb{E}_{y}\left(f\left(X_{s_{h}}^{h}\right)\right)-\mathbb{E}_{y}\left(f\left(X_{s_{h}}\right)\right)$.

There are two cases: either $d(y, \operatorname{supp} f)>d(\operatorname{suppf}, \partial D) / 4$ or $d(y, \operatorname{supp} f) \leq d(\operatorname{suppf}, \partial D) / 4$. In the first case introduce

$$
T_{f}=\inf \left\{s>0, X_{s} \in \operatorname{supp} f\right\} \quad \text { and } \quad T_{f}^{h}=\inf \left\{s>0, X_{s}^{h} \in \operatorname{supp} f\right\} .
$$

It is well known that there exists some constants $c$ and $c^{\prime}$ such that

$$
\mathbb{P}_{y}\left(T_{f}<s\right) \leq c^{\prime} e^{-c \frac{d^{2}(y, s u p p f)}{s}} .
$$

We claim that, provided $h<C d(\operatorname{supp} f, \partial D)$ for some sufficiently small constant $C$ only depending on $V, R, \sigma$ and the curvature of $\partial D$,

$$
\mathbb{P}_{y}\left(T_{f}<s\right) \leq c^{\prime} \frac{s}{h} e^{-c \frac{d^{2}(y, s u p p f)}{s}} .
$$

Some similar statement is shown for $T_{f}^{h}$ in Bossy et al. (2004). Actually this result is a consequence of the very general control of exit time for a one dimensional semi-martingale (see e.g. Ikeda and Watanabe (1981) lemma 8.5 p.342 or lemma 1 in Bossy et al. (2004)) which can be written for continuous or discrete time. The first thing to do is to introduce the continuous time version of the Euler scheme (see Bossy et al. (2004) p.883). Then if $d(y$, suppf $) \leq \frac{1}{2} d(\operatorname{suppf}, \partial D)$ we may simply replace $T_{f}^{h}$ by the smaller $T_{f}^{h} \wedge T_{\partial D}$ where $T_{\partial D}$ denotes the hitting time of the boundary and the result is a straightforward application of lemma 8.5 in Ikeda and Watanabe (1981). If $d(y$, supp $) \geq \frac{1}{2} d(\operatorname{supp} f, \partial D)$, one can feel the reflection term. But if $h<C d(\operatorname{suppf}, \partial D)$ as we said, the reflection will send the starting point at a distance less than $d(\operatorname{supp} f, \partial D) / 2$ of $\partial D$ up to a probability smaller than the probability for the norm of a standard gaussian vector to be larger than $C^{\prime} d(\operatorname{supp} f, \partial D) / 2 \sqrt{h}$ for some constant $C^{\prime}$, which is exactly the order we want. Hence, up to this error, the new starting point is again at a distance larger than $d(\operatorname{supp} f, \partial D) / 2$ of the support of $f$. This shows that the reflection when it occurs does not allow to get closer than $d(\operatorname{supp} f, \partial D) / 2$ of the support of $f$, up to an error of order $(s / h) e^{-c \frac{d^{2}(\partial D, \text { supp })}{s}}$, the pre-factor $s / h$ corresponding to the rough bound for the number of possible steps with reflection. Hence, up to this error, only the steps with no reflection can lead to enter the support of $f$. Of course in case of a huge gaussian increment the scheme does not move so does not enter the support of $f$ too.

As a consequence if $d(y, \operatorname{supp} f)>d(\operatorname{supp} f, \partial D) / 4$, for some new constants $c$ and $c^{\prime}$, it holds

$$
\begin{aligned}
\left|\mathbb{E}_{y}\left(f\left(X_{s_{h}}^{h}\right)\right)-\mathbb{E}_{y}\left(f\left(X_{s_{h}}\right)\right)\right| & \leq\left|\mathbb{E}_{y}\left(f\left(X_{s_{h}}^{h}\right)\right)\right|+\left|\mathbb{E}_{y}\left(f\left(X_{s_{h}}\right)\right)\right| \\
& \leq\|f\|_{\infty}\left(\mathbb{P}_{y}\left(T_{f}<s_{h}\right)+\mathbb{P}_{y}\left(T_{f}^{h}<s_{h}\right)\right) \\
& \leq c^{\prime} \frac{s_{h}}{h} e^{-c \frac{d^{2}(\partial D, s u p p f)}{s_{h}}}\|f\|_{\infty} .
\end{aligned}
$$


Now if $d(y$, supp $) \leq d(\operatorname{supp} f, \partial D) / 4$, we may replace $X$. and $X^{h}$. respectively by $Z$. and $Z^{h}$. before the exit time of $D$. A similar reasoning shows that

$$
\mathbb{P}_{y}\left(T_{\partial D}<s\right) \vee \mathbb{P}_{y}\left(T_{\partial D}^{h}<s\right) \leq c \frac{s}{h} e^{-c \frac{d^{2}(\partial D, \text { supp })}{s}}
$$

and since the same holds for the usual diffusion we finally get for $d(y, \operatorname{supp} f) \leq d(\operatorname{supp} f, \partial D) / 4$,

$$
\begin{aligned}
\left|\mathbb{E}_{y}\left(f\left(X_{s_{h}}^{h}\right)\right)-\mathbb{E}_{y}\left(f\left(X_{s_{h}}\right)\right)\right| & \leq\left|\mathbb{E}_{y}\left(f\left(Z_{s_{h}}\right)\right)-\mathbb{E}_{y}\left(f\left(Z_{s_{h}}^{h}\right)\right)\right|+ \\
& +c^{\prime} \frac{s_{h}}{h} e^{-c \frac{d^{2}(\partial D, \text { supp })}{s_{h}}}\|f\|_{\infty} .
\end{aligned}
$$

It remains to use Theorem 2.5 or (2.4). We thus obtain on one hand

$$
\begin{gathered}
\left|\mathbb{E}_{x}\left(f\left(X_{(N+1) s_{h}}^{h}\right)\right)-\mathbb{E}_{x}\left(f\left(X_{(N+1) s_{h}}\right)\right)\right| \leq \\
\left(C K\left(N s_{h}\right) h s_{h}^{-c(d)}+C(1) h+c^{\prime} \frac{s_{h}}{h} e^{-c \frac{d^{2}(\partial D, \text { supp })}{s_{h}}}\right)\|f\|_{\infty} .
\end{gathered}
$$

On the other hand$$
\left|\mathbb{E}_{x}\left(f\left(X_{(N+1) s_{h}}^{h}\right)\right)-\mathbb{E}_{x}\left(f\left(X_{(N+1) s_{h}}\right)\right)\right| \leq
$$$$
\leq C \sup _{(x, z) \in \bar{D} \times \bar{D}}\left\|p_{s_{h}}(., .)\right\|_{C_{b}^{6}(\bar{D} \times \bar{D})} K\left(N s_{h}\right) h\|F\|_{\infty}+c^{\prime} \frac{s_{h}}{h} e^{-c \frac{d^{2}(\partial D, \text { supp })}{s_{h}}}\|f\|_{\infty}+
$$$$
+h \frac{Q(1)}{s_{h}^{d q}}\left(\int P_{N_{h}}^{h}(x, d y) e^{-c(1) \frac{d^{2}(y, s u p p f)}{s_{h}}}\right)\|F\|_{\infty}
$$$$
\leq C \frac{h}{s_{h}^{c^{\prime}(d)}}\left(K\left(N s_{h}\right)+1\right)\|F\|_{\infty}+c^{\prime} \frac{s_{h}}{h} e^{-c \frac{d^{2}(\partial D, \text { supp })}{s_{h}}}\|f\|_{\infty} .
$$

We deduce from (2.7) that, provided

$$
s_{h} \leq \frac{2 c d^{2}(\operatorname{supp} f, \partial D)}{\ln (1 / h)},
$$

in such a way that

$$
e^{-c \frac{d^{2}(\operatorname{supp} f, \partial D)}{s_{h}}} \leq h^{2},
$$

there exist some constants $C$ and $K$ such that

$$
\left|\mathbb{E}_{x}\left(f\left(X_{N s_{h}}^{h}\right)\right)-\mathbb{E}_{x}\left(f\left(X_{N s_{h}}\right)\right)\right| \leq C h s_{h}^{-c(d)} K^{N}\|f\|_{\infty} .
$$

Of course we have to choose $s_{h}$ in such a way $s_{h} / h$ belongs to $\mathbb{N}$.

Hence we have obtained the first part of the Theorem, i.e.

$$
\begin{gathered}
\sup _{x \in \bar{D}}\left|\mathbb{E}_{x}\left(f\left(X_{T}^{h}\right)\right)-\mathbb{E}_{x}\left(f\left(X_{T}\right)\right)\right| \leq \\
\leq C h \ln ^{c(d)}(1 / h)(d(\operatorname{supp} f, \partial D))^{-2 c(d)} \exp \left(\frac{\theta \ln (1 / h) T}{d^{2}(\operatorname{supp} f, \partial D)}\right)\|f\|_{\infty} .
\end{gathered}
$$


This result is of course nor surprising, nor optimal. It just means that up to a time of order the square of the distance to the boundary, precisely $T_{h}=d^{2}(\operatorname{supp} f, \partial D) /(1+\varepsilon) \theta$, the reflected process and its Euler scheme do not feel the boundary, so that the evaluated quantity satisfies

$$
\sup _{x \in \bar{D}}\left|\mathbb{E}_{x}\left(f\left(X_{T_{h}}^{h}\right)\right)-\mathbb{E}_{x}\left(f\left(X_{T_{h}}\right)\right)\right| \leq C h^{\varepsilon /(1+\varepsilon)} \ln ^{c(d)}(1 / h)(d(\operatorname{supp} f, \partial D))^{-2 c(d)}\|f\|_{\infty} .
$$

But now we can use this result one more time writing again, this time for $t_{h} / h$ the integer part of the previous $T_{h} / h$,

$$
\begin{aligned}
& \qquad\left|\mathbb{E}_{x}\left(f\left(X_{(N+1) t_{h}}^{h}\right)\right)-\mathbb{E}_{x}\left(f\left(X_{(N+1) t_{h}}\right)\right)\right|= \\
& =\left|\int P_{N t_{h}}^{h}(x, d y)\left(\mathbb{E}_{y}\left(f\left(X_{t_{h}}^{h}\right)\right)-\mathbb{E}_{y}\left(f\left(X_{t_{h}}\right)\right)\right)+\int\left(P_{N t_{h}}^{h}(x, d y)-P_{N t_{h}}(x, d y)\right) \mathbb{E}_{y}\left(f\left(X_{t_{h}}\right)\right)\right| \\
& \leq \int P_{N t_{h}}^{h}(x, d y)\left|\mathbb{E}_{y}\left(f\left(X_{t_{h}}^{h}\right)\right)-\mathbb{E}_{y}\left(f\left(X_{t_{h}}\right)\right)\right|+K\left(N t_{h}\right) h\left\|\int f(z) p_{t_{h}}(., z) d z\right\|_{C_{b}^{5}(\bar{D})} \\
& \leq C\left(h^{\varepsilon /(1+\varepsilon)} \ln ^{c(d)}(1 / h)(d(\operatorname{supp} f, \partial D))^{-2 c(d)}+h K\left(N t_{h}\right)\left(1 \wedge t_{h}\right)^{-c(d)}\right)\|f\|_{\infty} \\
& \leq C(d(\operatorname{supp} f, \partial D))^{-2 c(d)}(1+\varepsilon)^{c(d)} h^{\varepsilon /(1+\varepsilon)} \ln ^{c(d)}(1 / h)\left(1 \vee K\left(N t_{h}\right)\right)\|f\|_{\infty} .
\end{aligned}
$$

Consequently, there exist positive constants $C$ and $\kappa^{\prime}$ such that for all smooth $f$ with compact support included in $D$, all $\varepsilon>0$ and all $T=N h$,

$$
\begin{gathered}
\sup _{x \in \bar{D}}\left|\mathbb{E}_{x}\left(f\left(X_{T}^{h}\right)\right)-\mathbb{E}_{x}\left(f\left(X_{T}\right)\right)\right| \leq \\
\leq C(d(\operatorname{supp} f, \partial D))^{-2 c(d)}(1+\varepsilon)^{c(d)} h^{\varepsilon /(1+\varepsilon)} \ln ^{c(d)}(1 / h) \exp \left(\frac{\kappa^{\prime}(1+\varepsilon) T}{d^{2}(\operatorname{supp} f, \partial D)}\right)\|f\|_{\infty} .
\end{gathered}
$$

If we write $\varepsilon=\alpha+\beta$ for some positive $\alpha$ and $\beta$, we may maximize

$$
h \mapsto h^{\alpha /(1+\varepsilon)} \ln ^{c(d)}(1 / h) \quad \text { for } h \in[0,1],
$$

yielding the second statement in the theorem.

The accurate reader remarked that at some places we introduced $\|F\|_{\infty}$, but that we were not able to eliminate all terms with $\|f\|_{\infty}$, even if they have some very small pre-factor. Actually we think that the absolutely continuous part of the law of the Euler scheme satisfies a result similar to Theorem 2.5, but do not succeed in proving it. Notice that a statement similar to our Theorem 2.6 is given in Gobet (2001b) Theorem 3.1. without proof.

\section{LONG TIME BEHAVIOUR.}

\subsection{Mixing for the reflected diffusion.}

If we write (1.6) in the form

$$
\inf _{x \in \bar{D}} P_{u}(x, d y) \geq \alpha_{u} m_{d}(\bar{D}) \frac{m_{d}(d y)}{m_{d}(\bar{D})},
$$


where $m_{d}$ denotes the Lebesgue measure, we recognize the so called Doeblin's condition. Doeblin's condition implies the following convergence result: let $\rho_{u}=1-\alpha_{u} m_{d}(\bar{D})$ which is of course positive and strictly less than 1 , then for $t>u$,

$$
\sup _{x \in \bar{D}}\left|P_{t}(x, d y)-\pi(d y)\right|_{T V} \leq 2 \rho_{u}^{t-u-1}
$$

Here $|\cdot|_{T V}$ denotes the total variation distance. The process is thus $\phi$-mixing (see, e.g., in Doukhan (1994), Section 2.4).

The main advantage of the previous derivation is that we have some explicit bound for the mixing rate.

For the aficionados of functional inequalities here is another proof of $\phi$-mixing. It is well known that for any smooth compact domain $\bar{D}$ the normalized Lebesgue measure $\lambda_{d}(d x)=$ $m_{d}(d x) / m_{d}(\bar{D})$ satisfies a Poincaré inequality

$$
\operatorname{Var}_{\lambda_{d}}(f) \leq C_{P}(\bar{D}) \int|\nabla f|^{2}(x) \lambda_{d}(d x)
$$

Since $\sigma$ is uniformly elliptic, we deduce that

$$
\operatorname{Var}_{\lambda_{d}}(f) \leq C_{P}(\bar{D}, \sigma) \int|\sigma \nabla f|^{2}(x) \lambda_{d}(d x) .
$$

Since $p_{i n v}$ is bounded from below and from above, Holley-Stroock classical perturbation argument shows that

$$
\operatorname{Var}_{\pi}(f) \leq C_{P}(\bar{D}, \sigma) \frac{\sup p_{i n v}}{\inf p_{i n v}} \int|\nabla f|^{2}(x) \pi(d x),
$$

from which we derive the exponential convergence

$$
\operatorname{Var}_{\pi}\left(P_{t} f\right) \leq e^{-C^{\prime}(\bar{D}, \sigma) t} \operatorname{Var}_{\pi}(f) .
$$

Of course one can come back to the $\phi$-mixing, using the following simple argument. Assume that $t \geq 1$ and that $f$ is bounded by 1 . Replacing $f$ by $f-\pi(f)$ we may assume that $f$ is $\pi$ centered but now bounded by 2 . Now, using the Markov property, we have

$$
\begin{aligned}
\left|\mathbb{E}_{x}\left(f\left(X_{t}\right)\right)\right| & =\left|\int p_{1}(x, z)\left(\int f(y) p_{t-1}(z, y) d y\right) d z\right| \\
& \leq \int \frac{p_{1}(x, z)}{p_{\text {inv }}(z)}\left|\left(\int f(y) p_{t-1}(z, y) d y\right)\right| \pi(d z)
\end{aligned}
$$

so that for $t \geq 1$,

$$
\sup _{x \in \bar{D}}\left|P_{t}(x, d y)-\pi(d y)\right|_{T V} \leq 2 \frac{\sup _{(x, y) \in \bar{D} \times \bar{D}} p_{1}(x, y)}{\inf p_{i n v}} e^{-C^{\prime}(\bar{D}, \sigma)(t-1) / 2} .
$$

In the sequel we shall use the following notation (similar to Shardlow et al. (2000)):

$$
\sup _{x \in \bar{D}}\left|P_{t}(x, d y)-\pi(d y)\right|_{T V} \leq C e^{-\omega t} .
$$




\subsection{Long time behavior of the symmetrized Euler scheme.}

We start this subsection by proving the Doeblin's condition for the Euler scheme. One way to get the Doeblin's condition is to derive a uniform in $x$ lower bound for the density $p_{t}^{h}(x,$.$) (whose$ total mass is less than one), since the singular part is a Dirac mass at $x$. Actually, despite the closeness of $p_{t}$ and $p_{t}^{h}$, this seems to be a difficult problem.

The first immediate lower bound is obtained for $p_{h}^{h}(x,$.$) i.e. the absolutely continuous part$ after one step, just taking into account the possible gaussian step and forgetting the possible reflection step. Hence thanks to uniform ellipticity we immediately have

$$
p_{h}^{h}(x, y) \geq C(\sigma) h^{-d / 2} \exp \left(-\frac{|\sigma(x)(y-x-b(x) h)|^{2}}{2 h}\right),
$$

so that using the boundedness of $b$ and $\sigma$ one can find constants depending on $\bar{D}, \sigma$ and $b$ such that

Hence

$$
\inf _{(x, y) \in \bar{D} \times \bar{D}} p_{h}^{h}(x, y) \geq c h^{-d / 2} e^{-C / h}=\alpha_{h}^{h} .
$$

$$
P_{h}^{h}(x, d y) \geq \alpha_{h}^{h} m_{d}(\bar{D}) \frac{m_{d}(d y)}{m_{d}(\bar{D})},
$$

and the Doeblin's condition is satisfied. We thus get first the existence of an invariant measure $\pi^{h}$ for the Euler scheme, second the fact that $\pi^{h}(d y)=p_{i n v}^{h}(y) d y$ thanks to $(2.1)$, since $P_{N h}^{h}(x, d y)$ converges in total variation to $\pi^{h}$ as $N$ goes to infinity, and finally the fact that the Euler scheme is $\phi$-mixing with rate $t \mapsto\left(1-\alpha_{h}^{h} m_{d}(\bar{D})\right)^{t}$. Of course this bound for the rate of convergence is disastrous for small $h$ 's.

One can think that for $h$ small enough the rate of convergence to equilibrium is more or less the same as for the reflected process. Actually and surprisingly, this result is quite difficult to prove rigorously, first of all because there is no result for the approximation of the densities of the Euler scheme by the ones of the original process. Hence to prove some exponential convergence uniform in $h$ we shall follow another way, the usual Meyn-Tweedie approach as suggested in Mattingly, Stuart and Higham (2002). Since we are in a compact situation, we shall actually follow the spirit of the method, and simplify the argument.

We will use the notations $Z$. and $Z^{h}$ as in the previous section for the non reflected diffusion process and Euler scheme, as well as $q$. and $q^{h}$ for their densities. Recall the following results which are classical or contained in Gobet and Labart (2008)

Lemma 3.2. There exist constants depending only on the dimension and the bounds of the coefficients and their derivatives such that for all $x$ and $y$ in $\mathbb{R}^{d}$, and all $s>0$,

$$
C_{1} s^{-d / 2} e^{-c_{1}|x-y|^{2} / s} \leq q_{s}(x, y) \leq C_{2} s^{-d / 2} e^{-c_{2}|x-y|^{2} / s},
$$

$$
q_{s}^{h}(x, y) \leq C_{3} s^{-d / 2} e^{-c_{3}|x-y|^{2} / s},
$$

$$
\left|q_{s}(x, y)-q_{s}^{h}(x, y)\right| \leq C_{4} h(1 \vee s) s^{-(d+1) / 2} e^{-c_{4}|x-y|^{2} / s} .
$$


1) is standard for elliptic diffusions, 2) is given in Gobet and Labart (2008) Proposition 3.5, 3) is Theorem 2.3 in Gobet and Labart (2008) (written here in a general form including small and large times).

We will use these estimates to get a "local" Doeblin condition. Recall that $R$ is defined at the beginning of Section 2 .

Lemma 3.3. Let $\varepsilon>0$ and $B \subset D$ be some closed ball of radius $\varepsilon$. Define $B_{\varepsilon}=\{z ; d(z, B) \leq$ $c \varepsilon\}$ for $c^{2}=2 c_{1} / c_{3}$. Assume that $d\left(B_{\varepsilon}, \partial D\right)>R$. Then there exist $h_{0}, \alpha_{0}$ and $s_{0} \leq 1$ such that for all $h \leq h_{0}$, and all $x \in B$,

$$
P_{\left[s_{0}\right]_{h}}^{h}(x, d y) \geq \alpha_{0} m_{d}(B) \mathbf{1}_{B}(y) \frac{m_{d}(d y)}{m_{d}(B)},
$$

where $[t]_{h}$ denotes the biggest number of the form $N$ h which is less than or equal to $t$.

Proof. Let $f$ be a smooth non-negative function supported by $B$. Denote by $T$ the first time the process $X^{h}$ exits $B_{\varepsilon}$. Here and in all what follows, all the considered times are of the form $N h$. Pick some $x \in B$ and $s \leq 1$. Then

$$
\mathbb{E}_{x}\left(f\left(X_{s}^{h}\right)\right) \geq \mathbb{E}_{x}\left(f\left(X_{s}^{h}\right) \mathbf{1}_{T>s}\right) \geq \mathbb{E}_{x}\left(f\left(Z_{s}^{h}\right) \mathbf{1}_{T>s}\right) .
$$

Notice that the final inequality is an inequality since the reflected Euler scheme stays at the initial point $x$ when the usual one performs a big jump.

For the usual Euler scheme we have

$$
\mathbb{E}_{x}\left(f\left(X_{s}^{h}\right) \mathbf{1}_{T>s}\right)=\mathbb{E}_{x}\left(f\left(X_{s}^{h}\right)\right)-\mathbb{E}_{x}\left(\mathbf{1}_{T \leq s} \mathbb{E}_{X_{T}^{h}}\left(f\left(X_{s-T}^{h}\right)\right)\right)
$$

so that

$$
\mathbb{E}_{x}\left(f\left(X_{s}^{h}\right) \mathbf{1}_{T>s}\right) \geq \int f(z) q_{s}^{h}(x, z) d z-\int f(z) \mathbb{E}_{x}\left(\mathbf{1}_{T \leq s} \sup _{y \notin B_{\varepsilon}} q_{s-T}^{h}(y, z)\right) d z .
$$

We thus have to bound from below

$$
a(x, z, h, s)=q_{s}^{h}(x, z)-\mathbb{E}_{x}\left(\mathbf{1}_{T \leq s} \sup _{y \notin B_{\varepsilon}} q_{s-T}^{h}(y, z)\right) .
$$

According to Lemma 3.2, we get

$$
\begin{aligned}
a(x, z, h, s) & \geq C_{1} s^{-d / 2} e^{-c_{1}|x-z|^{2} / s}-C_{4} h s^{-(d+1) / 2} e^{-c_{4}|x-z|^{2} / s}-\sup _{u \leq s} C_{3} u^{-d / 2} e^{-c_{3} c^{2} \varepsilon^{2} / u} \\
& \geq C_{1} s^{-d / 2} e^{-c_{1} \varepsilon^{2} / s}-C_{4} h s^{-(d+1) / 2}-\sup _{u \leq s} C_{3} u^{-d / 2} e^{-c_{3} c^{2} \varepsilon^{2} / u}
\end{aligned}
$$

Notice that if $s \leq c_{3} c^{2} \varepsilon^{2} /(d / 2)$, the supremum in the last term is achieved for $u=s$. Hence choosing $c^{2} c_{3}=2 c_{1}$ and $s \leq 2 c_{1} /(d / 2)$ we get

$$
C_{1} s^{-d / 2} e^{-c_{1}|x-z|^{2} / s}-\sup _{u \leq s} C_{3} u^{-d / 2} e^{-c_{3} c^{2} \varepsilon^{2} / u} \geq C_{1} s^{-d / 2} e^{-c_{1} \varepsilon^{2} / s}\left(1-\frac{C_{3}}{C_{1}} e^{-c_{1} \varepsilon^{2} / s}\right)
$$

so that choosing $s$ small enough i.e.

$$
s \leq s_{0}=\frac{4 c_{1} \varepsilon^{2}}{d} \wedge \frac{c_{1} \varepsilon^{2}}{\ln _{+}\left(2 C_{3} / C_{1}\right)},
$$

this term is larger than

$$
\frac{C_{1}}{2} s^{-d / 2} e^{-c_{1} \varepsilon^{2} / s}
$$


It remains to choose

and we get the result with

$$
h_{0}=\frac{1}{2} \sqrt{s_{0}} \frac{C_{1}}{2 C_{4}} e^{-c_{1} \varepsilon^{2} / s_{0}}
$$

$$
\alpha_{0}=\frac{C_{1}}{4}\left[s_{0}\right]_{h}^{-d / 2} e^{-c_{1} \varepsilon^{2} /\left[s_{0}\right]_{h}} .
$$

Let $x \in \bar{D}$. Using the Chapman-Kolmogorov relation we get

$$
P_{t+s}^{h}(x, d y) \geq \int P_{s}(z, d y) \mathbf{1}_{B}(z) P_{t}(x, d z) .
$$

Choose $s=\left[s_{0}\right]_{h}=t$. According to the previous lemma we get

$$
P_{2\left[s_{0}\right]_{h}}^{h}(x, d y) \geq P_{\left[s_{0}\right]_{h}}^{h}(x, B) \alpha_{0} m_{d}(B) \mathbf{1}_{B}(y) \frac{m_{d}(d y)}{m_{d}(B)} .
$$

But according to Theorem 2.6 with $f=\mathbf{1}_{B}$,

$$
P_{\left[s_{0}\right]_{h}}^{h}(x, B) \geq P_{\left[s_{0}\right]_{h}}(x, B)-C h^{\gamma}
$$

for some $\gamma>0$ and some constant $C$ that does not depend on $h$. Accordingly, thanks to (1.6), if $h$ is small enough

$$
\inf _{x \in \bar{D}} P_{\left[s_{0}\right]_{h}}^{h}(x, B) \geq \alpha_{0}^{\prime}>0 .
$$

We have thus proved that the following global Doeblin condition is satisfied: there exists a probability measure $\nu(d y)=\mathbf{1}_{B}(y) \frac{m_{d}(d y)}{m_{d}(B)}$ and a positive constant $c$ such that

$$
\inf _{x \in \bar{D}} P_{\left[s_{0}\right]_{h}}^{h}(x, d y) \geq c \nu(d y) .
$$

According to Doeblin's theorem we thus have obtained

Theorem 3.4. There exists $h_{1}>0, C>0$ and $\omega^{\prime}>0$ such that for all $N \in \mathbb{N}^{*}$, for all $h \leq h_{1}$,

$$
\sup _{x \in \bar{D}}\left|P_{N h}^{h}(x, d y)-\pi^{h}(d y)\right|_{T V} \leq C e^{-\omega^{\prime} N h} .
$$

We conclude this section by proving some results on the invariant measures using the beautiful argument in Shardlow and Stuart (2000) Theorem 3.3. with $\mathcal{G}_{0}$ therein given by the unit ball of $C_{b}^{5}(\bar{D})$ and $\bar{G}=1$. We get (recall that $\omega$ and $\kappa$ are defined respectively in (3.1) and proposition $2.3)$

Theorem 3.5. Let $T(h)=\frac{1}{\omega+2 \kappa} \ln (1 / h)$. Assume that $h$ is small enough for $T(h)$ to be larger than 1 and define $\gamma=\frac{\omega}{\omega+2 \kappa}$.

Then there exists some $C>0$ such that for all $N$ with $N h \geq T(h)$,

$$
\sup _{x \in \bar{D}}\left|\mathbb{E}_{x}\left(f\left(X_{N h}^{h}\right)\right)-\int f(y) \pi(d y)\right| \leq C h^{\gamma}\|f\|_{C_{b}^{5}(\bar{D})} .
$$

Consequently

$$
\left|\int f(y) \pi^{h}(d y)-\int f(y) \pi(d y)\right| \leq C h^{\gamma}\|f\|_{C_{b}^{5}(\bar{D})} .
$$

As a by-product we get the following 
Corollary 3.6. For all $t \geq 0$ it holds

$$
\sup _{x \in \bar{D}}\left|\mathbb{E}_{x}\left(f\left(X_{t}^{h}\right)\right)-\mathbb{E}_{x}\left(f\left(X_{t}\right)\right)\right| \leq C h^{\gamma}\|f\|_{C_{b}^{5}(\bar{D})} .
$$

Proof. Using homogeneity we may assume that $\|f\|_{C_{b}^{5}(\bar{D})}=1$.

For $t \leq T(h)$ we know from Theorem 2.2 and Proposition 2.3 that

$$
\left|\mathbb{E}_{x}\left(f\left(X_{t}^{h}\right)\right)-\mathbb{E}_{x}\left(f\left(X_{t}\right)\right)\right| \leq C e^{\kappa t} h \leq C e^{\kappa T(h)} h \leq C h^{\frac{\omega+\kappa}{\omega+2 \kappa}} \leq C h^{\gamma},
$$

provided $h<1$. For $t>T(h)$ we have according to the previous Theorem

$$
\left|\mathbb{E}_{x}\left(f\left(X_{t}^{h}\right)\right)-\int f(y) \pi(d y)\right| \leq C h^{\gamma}
$$

and thanks to $(3.1)$

$$
\left|\mathbb{E}_{x}\left(f\left(X_{t}\right)\right)-\int f(y) \pi(d y)\right| \leq C e^{-\omega t} \leq C e^{-\omega T(h)} \leq C h^{\gamma} .
$$

Similarly, choosing for $\mathcal{G}_{0}$ the set of bounded functions (bounded by 1 for instance), compactly supported in $D$ such that $d(\operatorname{supp} f, \partial D) \geq \eta>0$ to which we add the function $\bar{G}=1$, we may again apply Theorem 3.3 in Shardlow and Stuart (2000). To help the reader let us describe the exact relationship with the notation therein: their $\varepsilon$ is our $h, R_{1}$ and $\omega$ in assumption ACI are here $C$ and $\omega, R_{2}, \kappa$ and $s$ in assumption ACII are here respectively $\frac{C}{\alpha} \eta^{-2 c(d)}(1+\varepsilon)^{1+c(d)}$, $\kappa^{\prime}(1+\varepsilon) / \eta^{2}$ and $(\varepsilon-\alpha) /(\varepsilon+1)$. We thus get the following

Theorem 3.7. Let $\varepsilon>\alpha>0$ and $\eta>0$. Let

$$
T(h)=\frac{\varepsilon-\alpha}{(1+\varepsilon)\left(\omega+2 \kappa^{\prime}(1+\varepsilon) / \eta^{2}\right)} \ln (1 / h) .
$$

Assume that $h$ is small enough for $T(h)$ to be larger than 1 and define

$$
\gamma=\frac{\omega}{\omega+2 \kappa^{\prime}(1+\varepsilon) / \eta^{2}}
$$

Then there exists some $C>0$ such that for all $N$ with $N h \geq T(h)$, and all bounded function $f$ such that $d(\operatorname{supp} f, \partial D) \geq \eta$,

$$
\sup _{x \in \bar{D}}\left|\mathbb{E}_{x}\left(f\left(X_{N h}^{h}\right)\right)-\int f(y) \pi(d y)\right| \leq C\left(1+\frac{1}{\alpha} \eta^{-2 c(d)}(1+\varepsilon)^{1+c(d)}\right) h^{\gamma}\|f\|_{\infty} .
$$

As before it follows

Corollary 3.8. For all $N \geq 0$, all $f$ as in the previous theorem, it holds

$$
\sup _{x \in \bar{D}}\left|\mathbb{E}_{x}\left(f\left(X_{N h}^{h}\right)\right)-\mathbb{E}_{x}\left(f\left(X_{N h}\right)\right)\right| \leq C\left(1+\frac{1}{\alpha} \eta^{-2 c(d)}(1+\varepsilon)^{1+c(d)}\right) h^{\frac{\gamma(\varepsilon-\alpha)}{\varepsilon+1}}\|f\|_{\infty} .
$$

In particular for $N=+\infty$ we get

$$
\left|\int f(y) \pi^{h}(d y)-\int f(y) \pi(d y)\right| \leq C\left(1+\frac{1}{\alpha} \eta^{-2 c(d)}(1+\varepsilon)^{1+c(d)}\right) h^{\frac{\gamma(\varepsilon-\alpha)}{\varepsilon+1}}\|f\|_{\infty} .
$$




\section{Error estimation USing Euler scheme.}

In this section we will evaluate the $\mathbb{L}^{2}$ error made when replacing the reflected diffusion process by the symmetrized Euler scheme in the standard estimation procedures.

\subsection{Estimation of an observable.}

Consider some smooth function $f$ and its associated "observable" $\pi(f)=\int f d \pi$. A natural way to estimate $\pi(f)$ is to use the ergodic behavior of the process, hence to introduce

$$
\hat{\pi}_{n}(f)=\frac{1}{n} \sum_{i=1}^{n} f\left(X_{i h}\right)
$$

for some discretization step $h$. Using (3.1) we immediately have

$$
\sup _{x \in \bar{D}} \mathbb{E}_{x}\left[\left|\hat{\pi}_{n}(f)-\pi(f)\right|\right] \leq C \frac{1}{n\left(1-e^{-\omega h}\right)}\|f\|_{\infty} .
$$

Introducing $\tilde{f}=f-\pi(f)$, we also have

$$
\begin{aligned}
\mathbb{E}_{x}\left[\left|\hat{\pi}_{n}(f)-\pi(f)\right|^{2}\right] & =\frac{1}{n^{2}} \sum_{1 \leq i, j \leq n} \operatorname{Cov}_{x}\left(\tilde{f}\left(X_{i h}\right), \tilde{f}\left(X_{j h}\right)\right) \\
& \leq \frac{2}{n^{2}} \sum_{i \leq j=1}^{n} \mathbb{E}_{x}\left(\tilde{f}\left(X_{i h}\right) \mathbb{E}_{X_{i h}}\left(\tilde{f}\left(X_{(j-i) h}\right)\right)\right) \\
& \leq \frac{C\|f\|_{\infty}^{2}}{n^{2}} \sum_{i=1}^{n} \sum_{j \geq i}^{n} e^{-\omega(j-i) h}
\end{aligned}
$$

and finally

$$
\sup _{x \in \bar{D}} \mathbb{E}_{x}\left[\left|\hat{\pi}_{n}(f)-\pi(f)\right|^{2}\right] \leq C \frac{1}{n\left(1-e^{-\omega h}\right)}\|f\|_{\infty}^{2} .
$$

If we want to simulate or to predict the observable using the symmetric Euler scheme, we can replace $\hat{\pi}_{n}(f)$ by

$$
\hat{\pi}_{n}^{h}(f)=\frac{1}{n} \sum_{i=1}^{n} f\left(X_{i h}^{h}\right)
$$

Similarly we have that for $h$ small enough,

$$
\sup _{x \in \bar{D}} \mathbb{E}_{x}\left[\left|\hat{\pi}_{n}^{h}(f)-\pi^{h}(f)\right|^{2}\right] \leq C \frac{1}{n\left(1-e^{-\omega^{\prime} h}\right)}\|f\|_{\infty}^{2}
$$

In order to evaluate the least mean square error $\mathbb{E}_{x}\left[\left|\hat{\pi}_{n}^{h}(f)-\pi(f)\right|^{2}\right]$ we can introduce (add and subtract) $\pi^{h}(f)$. Using what precedes, Corollary 3.6 and Theorem 3.5 we then obtain

$$
\sup _{x \in \bar{D}} \mathbb{E}_{x}\left[\left|\hat{\pi}_{n}^{h}(f)-\pi(f)\right|^{2}\right] \leq C\left(\frac{1}{n\left(1-e^{-\omega^{\prime} h}\right)}\|f\|_{\infty}^{2}+h^{2 \gamma}\|f\|_{C_{b}^{5}}^{2}\right) .
$$

Equilibrating the two terms in the right hand side of Inequality (4.2) we obtain Theorem 4.3 below. 
Theorem 4.3. Let $f$ be some smooth function with associated observable $\pi(f)=\int_{D} f d \pi$. Let $\hat{\pi}_{n}(f)$ be defined as in (4.1). We get :

$$
\sup _{x \in \bar{D}} \mathbb{E}_{x}\left[\left|\hat{\pi}_{n}^{h}(f)-\pi(f)\right|^{2}\right] \leq C n^{\frac{-2 \gamma}{2 \gamma+1}},
$$

with $\gamma=\frac{\omega}{\omega+2 \kappa}$.

\subsection{Kernel estimation for the invariant density.}

Recall the definition of our kernel estimator introduced in (1.7):

$$
\hat{p}^{n, h}(y)=\frac{1}{n b_{n}^{d}} \sum_{i=1}^{n} K\left(\frac{y-X_{i h}^{h}}{b_{n}}\right)
$$

for some non-negative smooth kernel $K$ compactly supported in a neighborhood of the origin and such that $\int K(z) d z=1$. Additional conditions on $K$ will be introduced later. As we said in the introduction our goal is to get some control on $\mathbb{E}_{x}\left(\left|\hat{p}^{n, h}(y)-p_{\text {inv }}(y)\right|^{2}\right)$.

Of course we have

$$
\mathbb{E}_{x}\left(\left|\hat{p}^{n, h}(y)-p_{i n v}(y)\right|^{2}\right) \leq 2 \mathbb{E}_{x}\left(\left|\hat{p}^{n, h}(y)-\mathbb{E}_{\pi^{h}}\left(\hat{p}^{n, h}(y)\right)\right|^{2}\right)+2\left|\mathbb{E}_{\pi^{h}}\left(\hat{p}^{n, h}(y)\right)-p_{\text {inv }}(y)\right|^{2} .
$$

For the second term (the bias term) we have

$$
\begin{aligned}
\left|\mathbb{E}_{\pi^{h}}\left(\hat{p}^{n, h}(y)\right)-p_{\text {inv }}(y)\right|^{2}= & \left|\frac{1}{b_{n}^{d}} \int K\left(\frac{y-z}{b_{n}}\right) \pi^{h}(d z)-p_{\text {inv }}(y)\right|^{2} \\
\leq & 2\left|\frac{1}{b_{n}^{d}} \int K\left(\frac{y-z}{b_{n}}\right)\left(\pi^{h}(d z)-\pi(d z)\right)\right|^{2}+ \\
& +2\left|\frac{1}{b_{n}^{d}} \int K\left(\frac{y-z}{b_{n}}\right) \pi(d z)-p_{\text {inv }}(y)\right|^{2}
\end{aligned}
$$

On one hand, if we choose $K$ smooth and such that $\int P(u) K(u) d u=0$ for all polynomial with degree between 1 and $m-1$ it is classical that

$$
\left|\frac{1}{b_{n}^{d}} \int K\left(\frac{y-z}{b_{n}}\right) \pi(d z)-p_{i n v}(y)\right|^{2} \leq C b_{n}^{2 m} .
$$

On the other hand we may use Theorem 3.5 or Corollary 3.8 to get bounds for the first term, namely:

1) for all $y \in \bar{D}$,

$$
\left|\frac{1}{b_{n}^{d}} \int K\left(\frac{y-z}{b_{n}}\right)\left(\pi^{h}(d z)-\pi(d z)\right)\right|^{2} \leq C(K) \frac{h^{2 \gamma}}{b_{n}^{2(d+5)}}
$$

where $C(K)$ depends on the $C_{b}^{5}$ norm of $K$ and $\gamma=\frac{\omega}{\omega+2 \kappa}$,

2) for all $y \in D$,

$$
\left|\frac{1}{b_{n}^{d}} \int K\left(\frac{y-z}{b_{n}}\right)\left(\pi^{h}(d z)-\pi(d z)\right)\right|^{2} \leq C(K, d)\left(1+\left(d^{-1}(y, \partial D)\right)^{2 c(d)}\right)^{2} \frac{h^{2 \gamma^{\prime}}}{b_{n}^{2 d}},
$$


where $C(K, d)$ depends on $d$ and on the $\mathbb{L}^{\infty}$ norm of $K$, and for some constant $c(d)$ and $\gamma^{\prime}<\frac{1}{2} \frac{\omega}{\omega+4\left(\kappa^{\prime} / d^{2}(y, \partial D)\right)}($ we apply Corollary 3.8 with $\varepsilon=1)$.

It remains to control $A=\mathbb{E}_{x}\left(\left|\hat{p}^{n, h}(y)-\mathbb{E}_{\pi^{h}}\left(\hat{p}^{n, h}(y)\right)\right|^{2}\right)$.

Denote by $\tilde{K}(z)=K\left(\frac{y-z}{b_{n}}\right)-\int K\left(\frac{y-z}{b_{n}}\right) \pi^{h}(d z)$. We have, using the fact that $\tilde{K}$ is bounded by $2\|K\|_{\infty}$ and the control in Theorem 3.4,

$$
\begin{aligned}
n^{2} b_{n}^{2 d} A & =\sum_{i, j=1}^{n} \operatorname{Cov}_{x}\left(\tilde{K}\left(X_{i h}^{h}\right), \tilde{K}\left(X_{j h}^{h}\right)\right) \\
& \leq 2 \sum_{i \leq j=1}^{n} \mathbb{E}_{x}\left(\tilde{K}\left(X_{i h}^{h}\right) \mathbb{E}_{X_{i h}^{h}}\left(\tilde{K}\left(X_{(j-i) h}^{h}\right)\right)\right) \\
& \leq C\|K\|_{\infty}^{2} \sum_{i=1}^{n} \sum_{j \geq i}^{n} e^{-\omega^{\prime}(j-i) h}
\end{aligned}
$$

so that

$$
\mathbb{E}_{x}\left(\left|\hat{p}^{n, h}(y)-\mathbb{E}_{\pi^{h}}\left(\hat{p}^{n, h}(y)\right)\right|^{2}\right) \leq C \frac{1}{n b_{n}^{2 d}\left(1-e^{-\omega^{\prime} h}\right)}
$$

Let us gather all the results

Theorem 4.4. Let $K$ be some non-negative smooth kernel, compactly supported in a neighborhood of the origin such that $\int K(z) d z=1$ and $\int P(u) K(u) d u=0$ for all polynomial with degree between 1 and $m-1$.

Then there exist some $C$ depending on the domain, the coefficients and $K$, and some $H>0$ such that for all $h \leq H$, and all $(x, y) \in \bar{D} \times \bar{D}$,

$$
\mathbb{E}_{x}\left(\left|\hat{p}^{n, h}(y)-p_{\text {inv }}(y)\right|^{2}\right) \leq C E\left(n, h, b_{n}\right)
$$

where

- $\quad$ for $\gamma=\frac{\omega}{\omega+2 \kappa}$,

$$
E\left(n, h, b_{n}\right)=\max \left(\frac{1}{n b_{n}^{2 d}\left(1-e^{-\omega^{\prime} h}\right)}, b_{n}^{2 m}, \frac{h^{2 \gamma}}{b_{n}^{2(d+5)}}\right),
$$

- $\quad$ for $y \in D$ and $\gamma^{\prime}=\frac{\omega}{\omega+4\left(\kappa^{\prime} / d^{2}(y, \partial D)\right)}$,

$$
E\left(n, h, b_{n}\right)=\max \left(\frac{1}{n b_{n}^{2 d}\left(1-e^{-\omega^{\prime} h}\right)}, b_{n}^{2 m},\left(1+d^{-1}(y, \partial D)\right)^{c(d)} \frac{h^{2 \gamma^{\prime}}}{b_{n}^{2 d}}\right) .
$$




\section{NumericAl inVESTigations}

The goal of this section is twofold. On one hand one wants to illustrate the convergence of the estimator of the invariant density based on the Euler scheme. On the other hand, one raises the issue of tuning the parameters of the reflected Euler scheme, that is the initial point, the step $h$, and the parameter $R$ (see Section 2). We do not provide a theoretical answer to these issues, we rather show few simulations as a warning.

The model for $\left\{X_{t}=\left(X_{1, t}, X_{2, t}\right), t \geq 0\right\}$ we have chosen is driven by Equation (5.1) below:

$$
\begin{aligned}
& d X_{1, t}=d W_{1, t}-\beta \frac{X_{1, t}}{1+X_{1, t}^{2}+X_{2, t}^{2}} d t \\
& d X_{2, t}=d W_{2, t}-\beta \frac{X_{2, t}}{1+X_{1, t}^{2}+X_{2, t}^{2}} d t
\end{aligned}
$$

with $\beta>1, D=\left\{\left(x_{1}, x_{2}\right) \in \mathbb{R}^{2}, x_{1}^{2}+x_{2}^{2}<r\right\}, r>0$, and with the vector field defining the reflection direction normal to the boundary. The invariant density is then defined for $\mathbf{x}=$ $\left(x_{1}, x_{2}\right) \in D$ by Equation (5.2) below:

$$
p_{i n v}\left(x_{1}, x_{2}\right)=\frac{1-\beta}{\pi\left(2^{1-\beta}-1\right)} \frac{1}{\left(1+x_{1}^{2}+x_{2}^{2}\right)^{\beta}} .
$$

The invariant density is estimated with the kernel density estimator defined by (1.7) with $K(x, y)=\frac{1}{2 \pi} \exp ^{-\frac{x_{1}^{2}+x_{2}^{2}}{2}}, b_{n}=n^{-1 / 5}$ and [.] denoting the integer part. $\mathbf{X}_{0}^{h}=\left(X_{1,0}^{h}, X_{2,0}^{h}\right)$ is obtained by running the reflected Euler scheme from point $\left(x_{1}, x_{2}\right)$ and during $[n / 2]$ steps.

In the experiments below, we run the simulations for different values of $n, h,\left(x_{1}, x_{2}\right), R$ and $\beta$. Recall that if a particle is sent outside the $R$-neighbourhood of $\partial D$, that is is sent outside the region $\left\{\left(x_{1}, x_{2}\right) \in \mathbb{R}^{2}, x_{1}^{2}+x_{2}^{2} \leq r+R\right\}$, then the simulation is restarted. For each set of values of these parameters, we compute the mean integrated quadratic error of our estimation procedure, based on $K=30$ independent realizations, on a regular grid. More precisely, we consider a regular grid $\left(u_{j}, v_{j}\right)_{j=1, \ldots,(20 r+1)^{2}}$ of $[-r, r] \times[-r, r]$ with a step of size equal to 0.1 in each direction. We then define:

$$
e r r=\frac{1}{K} \sum_{k=1}^{K} \operatorname{err}_{k}=\frac{1}{K} \frac{1}{(20 r+1)^{2}} \sum_{j=1}^{(20 r+1)^{2}}\left(\hat{p}_{k}^{n, h}\left(u_{j}, v_{j}\right)-p_{i n v}\left(u_{j}, v_{j}\right)\right)^{2}
$$

where $\hat{p}_{k}^{n, h}, k=1, \ldots, K K$ independent realizations of our estimator.

The discretization of the theoretical invariant density, $p_{i n v}$, is drawn on Figure 2 for respectively $\beta=2$ and $\beta=1.1$. Then, for each experiment below, we provide two graphs representing the set of the 30 particles at the end of the simulation, and the estimated density.

First experiment: $\beta=2, r=4, R=0.1, h=0.05,\left(x_{1}, x_{2}\right)=(0,0), n=5.10^{4}$ (Figure 3).

Second experiment: $\beta=1.1, r=4, R=0.1, h=0.05,\left(x_{1}, x_{2}\right)=(0,0), n=5.10^{4}$ (Figure 4).

Third experiment: $\beta=2, r=2, R=0.1, h=0.05,\left(x_{1}, x_{2}\right)=(0,0), n=5.10^{4}$ (Figure 5). 

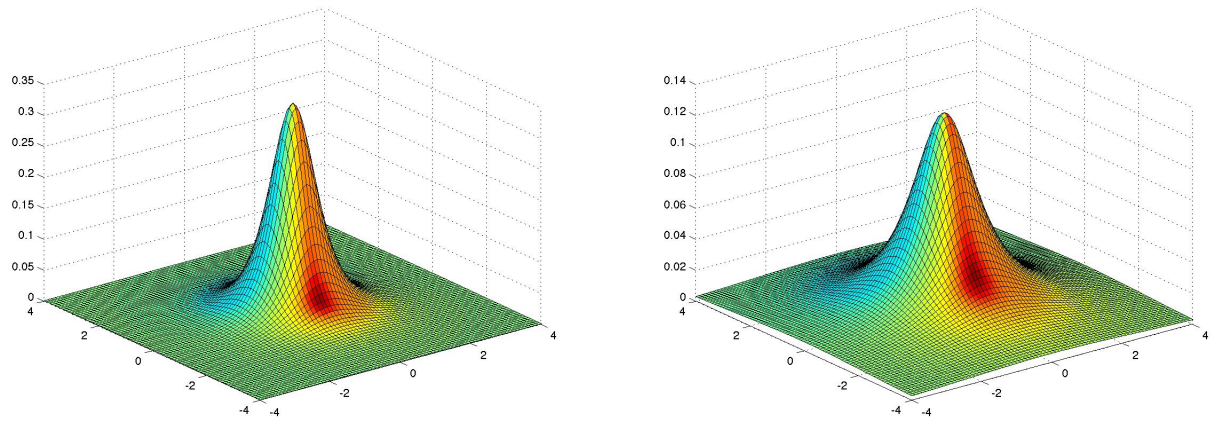

FiguRE 2. $p_{\text {inv }}$ with (left) $\beta=2$, (right) $\beta=1.1$
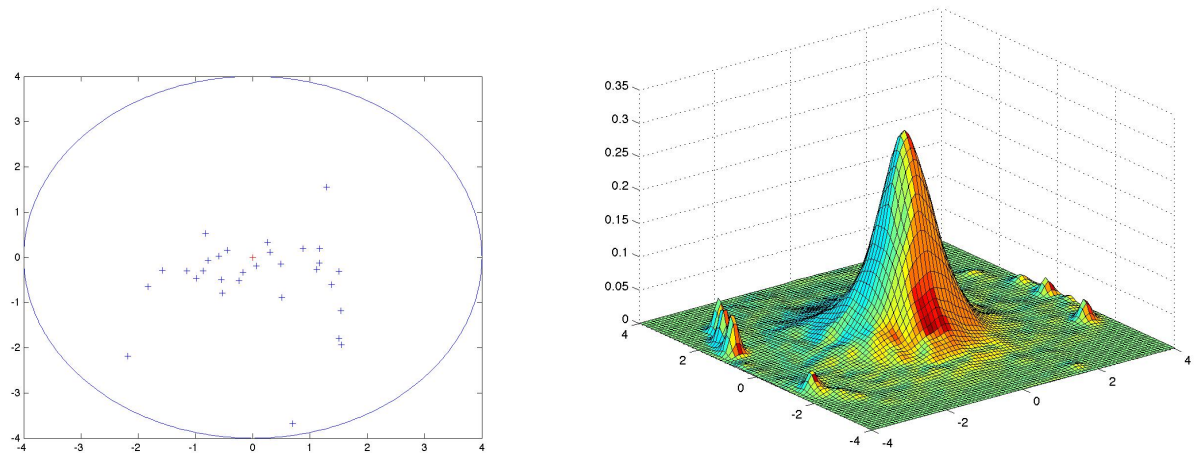

FiguRE 3. 1st experiment, (left) $K=30$ particles at the final time step, (right) $\hat{p}^{n, h}$
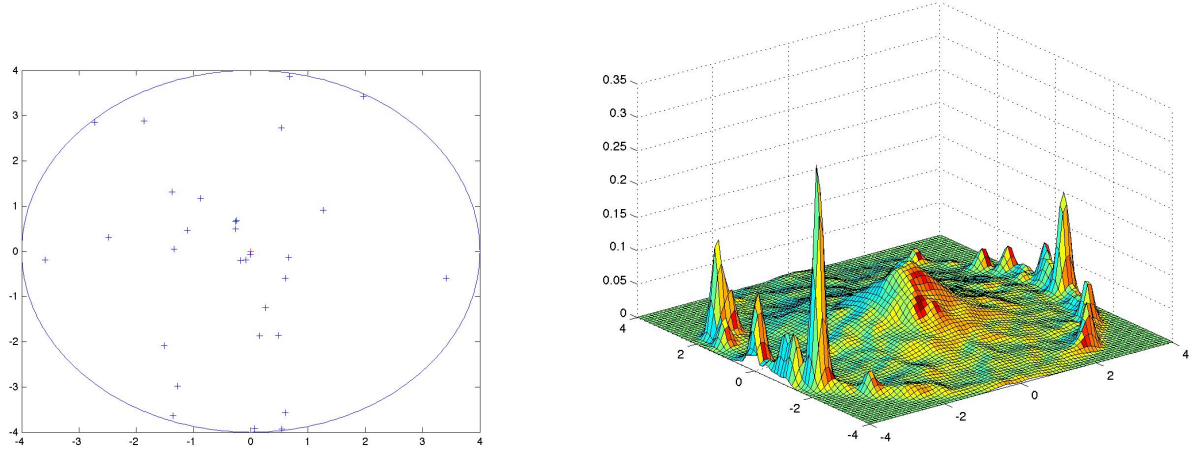

FiguRE 4. 2nd experiment, (left) $K=30$ particles at the final time step, (right) $\hat{p}^{n, h}$

Fourth experiment: $\beta=2, r=4, R=0.1, h=0.05,\left(x_{1}, x_{2}\right)=(1,0.5), n=5.10^{4}$ (Figure 6).

Fith experiment: $\beta=2, r=4, R=0.1, h=0.1,\left(x_{1}, x_{2}\right)=(0,0), n=5.10^{4}$ (Figure 7). 

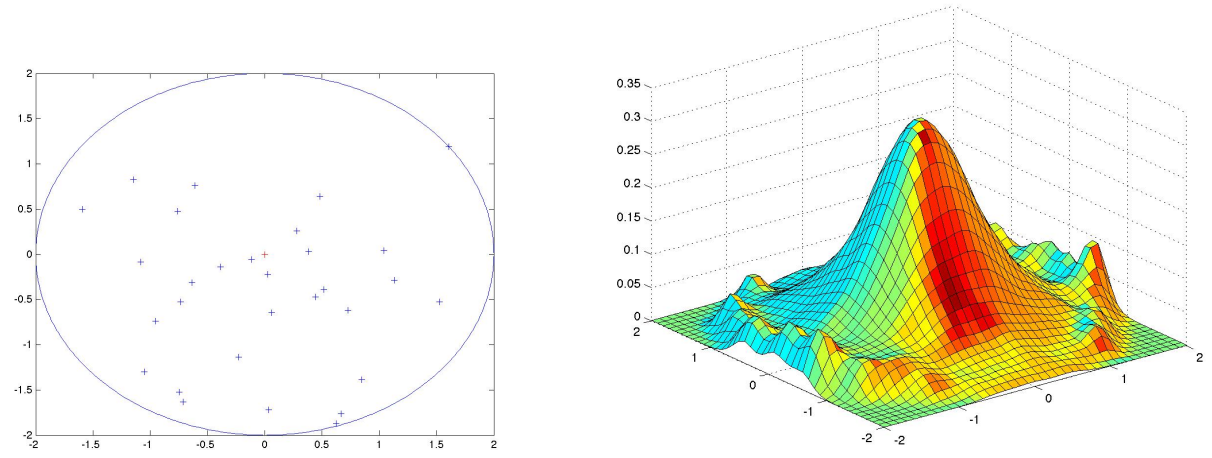

FiguRE 5. 3rd experiment, (left) $K=30$ particles at the final time step, (right) $\hat{p}^{n, h}$
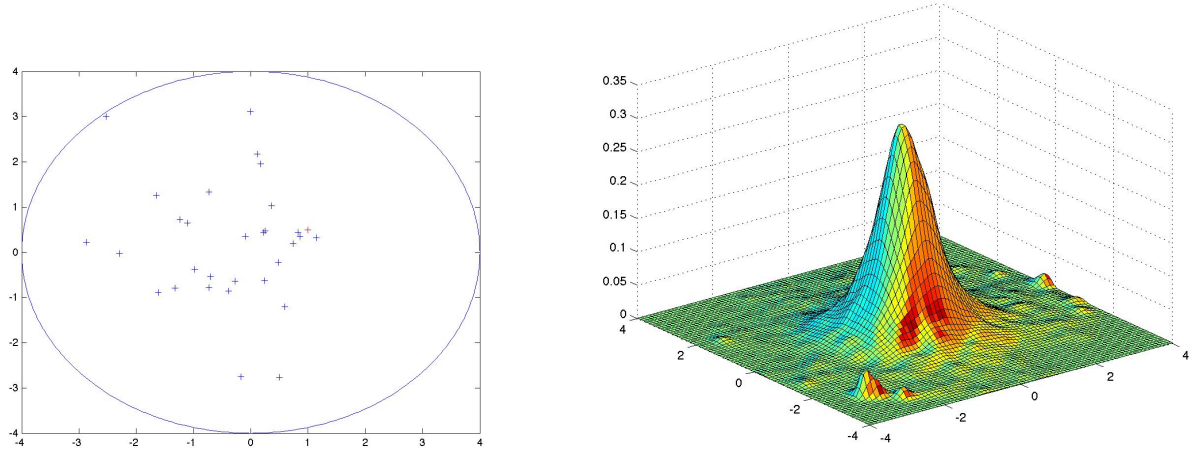

FiguRE 6. 4th experiment, (left) $K=30$ particles at the final time step, (right) $\hat{p}^{n, h}$
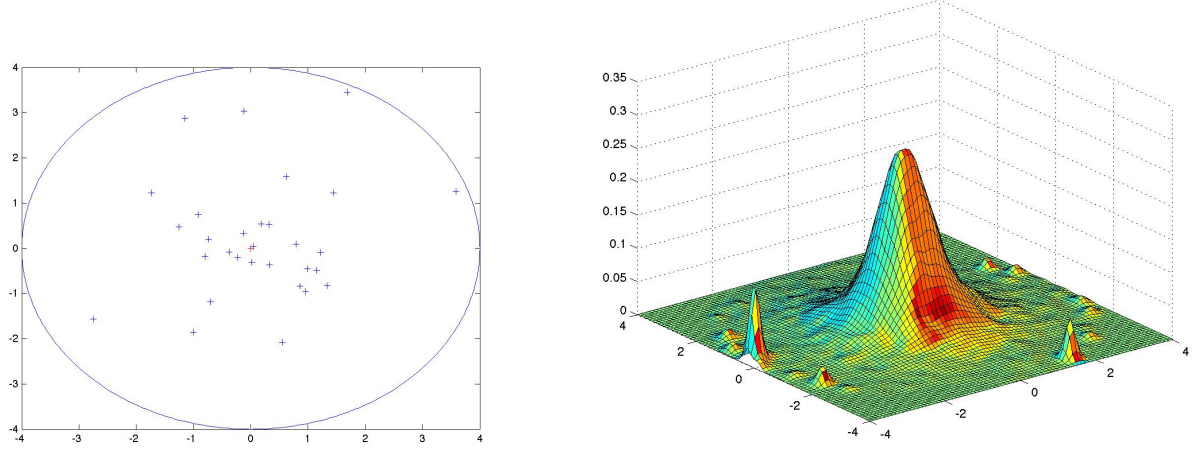

FiguRE 7. 4 th experiment, (left) $K=30$ particles at the final time step, (right) $\hat{p}^{n, h}$ 
The conclusion of these five experiments, all realized with $n=5 \cdot 10^{4}$, is that the most influent parameters are the ratio $r / R$ (see Figure 5) and the value of $\beta$ in the equations driving the model (see Equations (5.1) and Figure 4). The invariant probability measure with $\beta=1.1$ (Figure 2 on the right) has heavier tails than the one with $\beta=2$ (Figure 2 on the right). On Figure 4 we see that the scheme has difficulties to escape from the inside boundary of the disc. It seems a rather intricate issue to get an analytical expression for the rate of convergence of our estimate with respect to these parameters. The choice of the initial point $\left(x_{1}, x_{2}\right)$, of the step $h$ seems less important (see Figures 6 and 7).

We now study the convergence of our estimate, with parameters fixed as in the fisrt experiment, except $n$ which varies from $n=5.10^{2}, 5.10^{3}$ and $5.10^{4}$. For each value of $n$ we compute the mean integrated square error defined by (5.3). The results are stated in Table 1.

\begin{tabular}{|c|c|c|c|}
\hline$n$ & 500 & 5000 & 50000 \\
\hline error & $5.4933 \mathrm{e}-04$ & $1.7677 \mathrm{e}-04$ & $3.0297 \mathrm{e}-05$ \\
\hline
\end{tabular}

TABLE 1. Evolution of the mean integrated squared error for $n=5.10^{2}, 5.10^{3}$ and $5.10^{4}$ with $\beta=2, r=4, R=0.1, h=0.05,\left(x_{1}, x_{2}\right)=(0,0)$. The error is computed on $K=30$ independent realizations.

\section{Overall conclusions}

The problem of approaching the invariant measure of an ergodic diffusion is an important problem with many applications in the simulation of natural processes. As a paradigmatic example, we can cite the Langevin method to simulate a random sample of a random variable in $\mathbb{R}^{d}$ having a given density. The procedure is also used in molecular dynamics, neuronal modeling, environmental studies, etc. Usually the true system is replaced by an approximating numerical scheme, for instance the Euler scheme or any other. The main issue in such a study is that the global time of the scheme must be chosen sufficiently large for approaching well the invariant measure. This latter fact makes difficult to evaluate the error of approximation. In the present work we have tackled the case when the system under study is a reflected diffusion in a smooth domain of $\mathbb{R}^{d}$. As it is well known, the reflection introduces intricacies in the control of the Euler scheme, while the compactness of the domain helps in the control of densities. We have obtained a bound for the error in the case of approaching an observable i.e. $\pi(f)$ (this case has been already studied for other systems by different authors, see for instance Talay (1990) and more recently the article of Honoré et al. (2016) where the approximation is obtained via a recursive algorithm) but we also consider, for the first time, the problem of approaching the density of the invariant measure. The obtained bounds are not optimal and at this stage, they do not allow to establish other interesting results as for example a central limit theorem for the estimators. Ergodic diffusions in unbounded domains can be similarly studied, this time with better controls on the numerical scheme, but with a more careful study of their ergodic properties. To complete and extend the results of this work will be the goal of further studies. 
Acknowledgments. This work has been partially supported by the project MATH-AmSud SIDRE Statistical inference for dependent stochastic processes and application in renewable energy and by the Inria International Chairs program.

\section{REFERENCES}

V. Bally and D. Talay (1995). The law of Euler scheme for stochastic differential equations (I): Convergence rate of the distribution function. Probab. Theory and Rel. Fields 104 (1), p. 43-60.

V. Bally and D. Talay (1996). The law of Euler scheme for stochastic differential equations (II): Convergence rate of the density. Monte Carlo Methods Appl. 2 (2), p. 93-128.

M. Bossy, E. Gobet and D. Talay (2004). A symmetrized Euler scheme for an efficient approximation of reflected diffusions. J. Appl. Prob. 41, p. 877-889.

P. Cattiaux (1992). Stochastic calculus and degenerate boundary value problems. Ann. Institut Fourier 42 (3), p. 541-624.

P. Cattiaux, J. R. Leon and C. Prieur (2014). Estimation for stochastic damping Hamiltonian systems under partial observations: I. Invariant density. Stoch. Proc. and their Appli. 124 (3), p. 1236-1260.

J. Dedecker and C. Prieur (2005). New dependence coefficients. Examples and applications to statistics. Probab. Theory and Relat. Fields 132, p. 203-236.

P. Doukhan (1994). Mixing. Properties and examples. Lecture Notes in Statistics, 85. Springer-Verlag, New York, 1994.

E. Gobet (2001a). Euler schemes and half-space approximation for the simulation of diffusions in a domain. ESAIM Prob. Stat. 5, p. 261-297.

E. Gobet (2001b). Efficient schemes for the weak approximation of reflected diffusions. Monte Carlo Methods Appl. 7 (1-2), p. 193-202.

E. Gobet and C. Labart (2008). Sharp estimates for the convergence of the density of the Euler scheme in small time. Elec. Comm. in Prob. 13, p. 352-363.

J. Guyon (2006). Euler schemes and tempered distributions. Stoch. Proc. and their Appli. 116, p. $877-904$.

I. Honoré, S. Menozzi and G. Pagès (2016). Non asymptotic Gaussian estimates for the recursive approximation of the invariant measure of a diffusion. arXiv 1605.08525v1. 
N. Ikeda and S. Watanabe (1981). Stochastic differential equations and diffusion processes. North Holland.

J.C. Mattingly, A.M. Stuart and D.J. Higham (2002). Ergodicity for SDEs and approximations: locally Lipschitz vector fields and degenerate noise. Stoch. Proc. and their Appli. 101, p. 185-232.

S. Meyn and R. Tweedie (2009). Markov chains and stochastic stability. Second edition. With a prologue by Peter W. Glynn. Cambridge University Press, Cambridge.

T. Shardlow and A.M. Stuart (2000). A perturbation theory for ergodic Markov chains and application to numerical approximations. SIAM J. Numer. Anal. 37 (4), p. 1120-1137.

D. Talay (1990). Second order discretization schemes of stochastic differential systems for the computation of the invariant law. Stochastics and Stochastic Reports 29 (1), p. 13-36.

M. Valera-López, A. Pineda and J.R. León (2016). Application of satellite image to the implementation of two stochastic models for modeling the transport of chlorophyll-a on Lake Valencia (Venezuela). Technical report, submitted, HAL Id : tel-01293471, version 2.

Patrick CattiauX, Institut de Mathématiques de Toulouse. Université de Toulouse., CnRS UMR 5219.,, 118 route de Narbonne, 31062 Toulouse Cedex 09, France.

E-mail address: cattiaux@math.univ-toulouse.fr

José LeÓn, Escuela de Matemática, Facultad de Ciencias, Universidad Central de Venezuela, Av. Los Ilustres, Los Chaguaramos, Caracas 1040, Venezuela.

E-mail address: jose.leon@ciens.ucv.ve

Clémentine PRIEUR, Université Grenoble Alpes, CnRS Laboratoire Jean Kuntzmann, Airsea Inria project/team, Grenoble Cedex, France.

E-mail address: clementine.prieur@imag.fr 